\title{
DIQUAT E UREIA NO MANEJO DA FLORAÇÃO NATURAL DO ABACAXIZEIRO 'PÉROLA'1
}

\author{
HELIO GASPAR KUOOS KIST ${ }^{2}$, JOSÉ DARLAN RAMOS ${ }^{3}$, RAFAEL PIO ${ }^{4}$, \\ VERÔNICA ANDRADE DOS SANTOS ${ }^{5}$
}

RESUMO - O florescimento natural do abacaxizeiro constitui-se num dos problemas mais sérios do seu manejo, devido à desuniformização e colheita em épocas impróprias, o que eleva o custo de produção e reduz o preço de venda. O objetivo deste trabalho foi avaliar o comportamento do diquat e da ureia como fitorreguladores no retardamento da diferenciação floral do abacaxizeiro cv. Pérola. Foram testadas as concentrações de $0 ; 15 \mathrm{mg} \mathrm{L}^{-1} ; 23 \mathrm{mg} \mathrm{L}^{-1}$; e $30 \mathrm{mg} \mathrm{L}^{-1}$ de diquat, sem e com ureia $\left(20 \mathrm{~g} \mathrm{~L}^{-1}\right)$, aplicando $50 \mathrm{~mL}^{-1}$ de calda sobre as folhas do abacaxizeiro uma única vez. O delineamento utilizado foi o de blocos casualizados, com quatro repetições. Avaliaram-se a inibição da floração natural em três datas, a massa dos frutos, a massa da coroa, a massa dos filhotes e o número de filhotes por planta, o comprimento e a largura da folha " $D$ " e os sólidos solúveis ( ${ }^{\circ}$ Brix). Os resultados mostraram que a aplicação de $30 \mathrm{ml} \mathrm{L}^{-1}$ de diquat retardou a floração natural, mas causou redução na massa dos frutos. $\mathrm{O}$ tratamento de $23 \mathrm{ml} \mathrm{L}^{-1}$ com ureia também determinou uma redução na floração natural, sem, no entanto, causar diminuição dos frutos. As doses de diquat não interferiram na massa e na produção de filhotes, no comprimento da folha " $\mathrm{D}$ " e no teor de sólidos solúveis ( ${ }^{\circ}$ Brix) do suco dos frutos. A ureia potencializou a ação do diquat no retardamento da floração.

Termos para indexação: Ananas comosus. Controle da floração. Práticas culturais. Cerrado Matogrossense.

\section{DIQUAT AND UREA IN THE NATURAL FLOWERING MANAGEMENT OF 'PEROLA' PINEAPPLE}

\begin{abstract}
The natural flowering of the pineapple plant is constituted in one of the most serious problems of its management, due to desuniformization and harvest in unsuitable times, lifting up the cost of production and reduction of the sale price. The objective of this study was to evaluate the behavior of diquat and urea as growth regulators in delaying floral induction of pineapple cv. Pearl. It was tested concentrations of $0 ; 15 \mathrm{mg} \mathrm{L}^{-1} ; 23 \mathrm{mg} \mathrm{L}^{-1} ; 30 \mathrm{mg} \mathrm{L}^{-1}$ of diquat, with and without urea $\left(20 \mathrm{~g} \mathrm{~L}^{-1}\right)$, using $50 \mathrm{~mL}^{-1}$ syrup over the leaves of pineapple only once. The experimental design was randomized blocks with four repetitions. It was evaluated the inhibition of natural flowering in three dates, the mass of fruits, the mass of the crown, the mass of the offspring and the number of offspring per plant, the length and width of leaves " $\mathrm{D}$ " and soluble solids ( ${ }^{\circ}$ Brix). The results showed that the application of $30 \mathrm{ml} \mathrm{L}^{-1}$ diquat have delayed the natural flowering, but caused reduction in the fruit mass. The treatment of $23 \mathrm{ml} \mathrm{L}^{-1}$ with urea also established a reduction in flowering natural, without; however, cause reduction in the fruit size. The doses of diquat did not interfere in the mass and production of offspring, length of leaf " $\mathrm{D}$ " and the content of soluble solids ( ${ }^{\circ}$ Brix) of the fruit juice. The urea potentiated the action of diquat in delaying the natural flowering.
\end{abstract}

Index terms: Ananas comosus, control of flowering, cultural practices, cerrado Matogrossense.

\footnotetext{
'(Trabalho 084-11). Recebido em: 07-03-2011. Aceito para publicação em: 12-07-2011. Parte da tese de doutorado do primeiro autor, apresentada à Universidade Federal de Lavras em 08/12/2010.

${ }^{2}$ Eng. ${ }^{\circ}$ Agrônomo, D.Sc., Pesquisador da Empresa Matogrossense de Pesquisa, Assistência e Extensão Rural (EMPAER-MT), Rua José Alves de Souza, 52 CEP 78300-000, Tangará da Serra-MT E-mail: heliokist@yahoo.com.br

${ }^{3}$ Eng. ${ }^{\circ}$ Agrônomo, D.Sc., Professor da Universidade Federal de Lavras-MG, Caixa Postal 3037 - CEP 37200-000, E-mail: darlan@dag.ufla.br.

${ }^{4}$ Eng. ${ }^{\circ}$ Agrônomo, D.Sc., Professor da Universidade Federal de Lavras-MG, Caixa Postal 3037 - CEP 37200-000, E-mail: rafaelpio@dag.ufla.br ${ }^{5}$ Eng. ${ }^{a}$ Agrônoma, M.Sc., Universidade Federal de Lavras-MG, Caixa Postal 3037 - CEP 37200-000 E-mail: veronicaandrad@yahoo.com.br
} 


\section{INTRODUÇÃO}

A uniformidade do florescimento e do amadurecimento dos frutos de abacaxi em um plantio comercial são aspectos de fundamental importância na busca de sucesso nesta atividade por facilitarem sobremaneira a colheita, o controle de pragas e doenças e a comercialização. Análises de mercado demonstraram que, entre os anos de 2003 a 2007, o preço médio do abacaxi, nos meses de escassez de oferta, fevereiro a abril, gira em torno de $\mathrm{R} \$ 1,50$, enquanto nos meses de novembro e dezembro, quando há oferta abundante, os preços médios sofrem uma acentuada queda para $\mathrm{R} \$ 1,25$ (AGRIANUAL, 2009).

A colheita e a comercialização nas épocas mais favoráveis de preços são viabilizadas pela utilização de indutores florais, como o ethefon, que além da uniformização permitem também a antecipação da diferenciação floral, produzindo comercialmente de forma racional e econômica.

Ocorre, entretanto, que, em algumas situações, o retardamento do florescimento seria mais conveniente que a antecipação, visando especialmente a ampliar o período de safra e deslocá-la para épocas mais favoráveis à comercialização. Uma das possibilidades seria através do emprego de alguns fitorreguladores, mas, para isso, é imprescindível o conhecimento do ciclo natural do abacaxizeiro nas diversas regiões produtoras. Os diversos fatores ambientais envolvidos na diferenciação floral natural estão relacionados ao encurtamento do dia, à baixa temperatura, principalmente a noturna, e também à redução da insolação devido a nebulosidade. Há evidências de que a baixa temperatura noturna aumenta o nível de atividade da auxina livre na planta, o que causa o florescimento, reduzindo assim a exigência de dias curtos (CUNHA et al.,1999).

A substância que tem proporcionado os melhores resultados na inibição do florescimento, é o Paclobutrazol. Foram obtidas percentagens superiores a $90 \%$ de inibição com aplicações de 150 e $200 \mathrm{mg} \mathrm{L}^{-1}$ do Paclobutrazol, e de de $82,22 \%$ quando utilizado a $100 \mathrm{mg} \mathrm{L}^{-1}$ (ANTUNES et al., 2008; BARBOSA et al., 1998). A inibição da produção do etileno pelo Paclobutrazol interfere na diferenciação floral do abacaxi. Uma alta concentração de ACC oxidase (1-aminociclopropano-1-carboxílico) no estádio reprodutivo do abacaxizeiro pode indicar que o etileno seja um dos fatores envolvidos na indução floral. Wang e Steffens (1985) afirmam que a conversão de ACC a etileno é determinada por inibidores vegetais.

O Fruitone CPA (ácido 2-3 clorofenoxi- propiônico), nas concentrações de 90 a $240 \mathrm{mg} \mathrm{L}^{-1}$, inibiu, reduziu ou retardou a floração natural na cv. Pérola, enquanto no Smooth Cayenne houve uma inibição de 76 e $82 \%$ em densidades de 33.000 e 46.000 plantas/há, respectivamente (CUNHA et al., 2003; REBOLLETO et al., 1997). O ácido 2-(3-clorofenoxi) propiônico não respondeu significativamente na floração da cv. 'Pérola'. mas pulverizações com 50 e $100 \mathrm{mg} \mathrm{L}^{-1}$ determinaram alterações internas e externas nas folhas, apresentando efeito fitotóxico (BARBOSA et.al. 1998; RABIE et al., 2000; BARBOSA et al., 2003).

Outros produtos, como o cloreto de mepiquat, ANA, $\mathrm{GA}_{3}$, tebuconazole e propiconazole foram testados, mas não apresentaram efeito sobre o florescimento natural (BARBOSA et al. , 1998; SAMPAIO et al., 1998; CUNHA et al., 2003).

Nickell (1982), trabalhando com a cultura da cana-de-açúcar, conseguiu controlar $100 \%$ da floração, aplicando $14 \mathrm{~g}$ de diquat $\mathrm{ha}^{-1}$. Portanto, este, além de ser um herbicida de contato, funciona também como fitorregulador vegetal, quando empregado em baixas concentrações, na ordem de $\mathrm{mg} /$ $\mathrm{kg}$. Este produto já vem sendo usado comercialmente como inibidor da floração em cana-de-açúcar. No abacaxizeiro, não há relato de pesquisas na literatura consultada.

Assim, o objetivo deste trabalho foi avaliar o comportamento do diquat e da ureia como fitorreguladores no retardamento da diferenciação floral do abacaxizeiro cv. Pérola, visando a redirecionar a colheita para períodos mais favoráveis a comercialização, e também obter melhor escalonamento da produção, ampliando o período de colheita.

\section{MATERIAL E MÉTODOS}

O experimento foi conduzido no período de abril de 2007 a junho de 2008, no campo experimen-

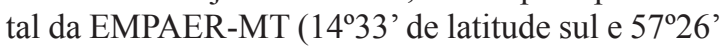
a oeste), no município de Tangará da Serra-MT. O solo onde foi implantado o experimento foi classificado por Orioli (1985) como Terra Roxa Estruturada Eutrófica, atual Nitossolo Verelho eutroférrrico. O clima, pela classificação de Köeppen, enquadra-se como Tropical Am. Dados climáticos são apresentados na Tabela 1 .

O plantio foi realizado em abril de 2007 , utilizando-se de mudas do tipo filhote da cultivar Pérola, com 25 a $30 \mathrm{~cm}$ de altura, em fileiras duplas, espaçadas de $0,90 \mathrm{~m} \times 0,40 \mathrm{~m} \times 0,30 \mathrm{~m}$. Os tratos culturais foram efetuados conforme recomendações para a cultura, e as adubações, baseadas em Souza et al.(1999), sem irrigação. 
O delineamento experimental foi o de blocos casualizados, com quatro repetições e sete tratamentos, equivalentes as concentrações de $0 ; 15 \mathrm{mg} \mathrm{L}^{-1}$ diquat; $15 \mathrm{mg} \mathrm{L}^{-1}$ diquat $+20 \mathrm{~g} \mathrm{~L}^{-1}$ de ureia; $23 \mathrm{mg} \mathrm{L}^{-1}$ diquat; $23 \mathrm{mg} \mathrm{L}^{-1}$ diquat $+20 \mathrm{~g} \mathrm{~L}^{-1}$ de ureia; $30 \mathrm{mg} \mathrm{L}^{-1}$ de diquat; $30 \mathrm{mg} \mathrm{L}^{-1}$ de diquat $+20 \mathrm{~g} \mathrm{~L}^{-1}$ de ureia. O produto comercial utilizado foi o Reglone ${ }^{\circledR}\left(200 \mathrm{~g} \mathrm{~L}^{-1}\right.$ de diquat). Adicionou-se à calda, o espalhante adesivo Agrex (ácido dodecilbenzeno - ácido sulfônico de concentração $50 \mathrm{~g} \mathrm{~L}^{-1}$ ). Foram aplicados $50 \mathrm{~mL}^{-1} \mathrm{da}$ calda sobre as folhas das plantas. As parcelas eram constituídas de 180 plantas, sendo as 120 centrais consideradas úteis. $\mathrm{O}$ tamanho das parcelas foi de $3,5 \mathrm{~m} \times 9,0 \mathrm{~m}$.

A aplicação dos tratamentos ocorreu em 16 de abril de 2008, aos 12 meses após o plantio, através de jato dirigido às folhas com pulverizador costal. Neste momento, as plantas ainda não apresentavam a inflorescência porque as condições climáticas responsáveis pelo processo de diferenciação floral (temperatura, fotoperíodo, precipitação) ainda não eram suficientemente favoráveis, muito embora os abacaxizeiros já houvessem atingido um nível de crescimento que lhes confere condições para absorver os estímulos florais naturais. A queda das temperaturas e a precipitação (Tabela 1) só começaram a ocorrer após a aplicação dos tratamentos. As avaliações da percentagem de floração foram realizadas em 20 de agosto, 15 de setembro e 05 de outubro, quando era observado o centro da roseta foliar e anotadas as plantas onde se podia visualizar a inflorescência. $\mathrm{O}$ cálculo de inibição foi realizado pela fórmula [(x - y) x 100] / x em que o " $\mathrm{x}$ " representa o número total de plantas dentro da parcela do tratamento que efetivamente participaram do teste, ou seja, foram descontadas das 120 plantas úteis constituintes da parcela, aquelas que já apresentavam a inflorescência na data de aplicação dos tratamentos, as que se encontravam atacadas por pragas, doenças, injúrias mecânicas e aquelas que foram induzidas artificialmente em novembro, após o encerramento das avaliações e, mesmo assim, não floresceram; o " $y$ " representa o número de plantas que floresceram até a data da respectiva avaliação. Foram também avaliados a massa dos frutos, a massa da coroa, a massa dos filhotes, o número de filhotes por planta, o comprimento e a largura da folha " $D$ ", e os sólidos solúveis ( ${ }^{\circ}$ Brix), com o intuito de detectar se ocorreu alguma anomalia nesses parâmetros em decorrência da aplicação dos tratamentos.

A análise estatística foi realizada, utilizandose do programa Sisvar. Os resultados foram submetidos à análise da variância através do teste $\mathrm{F}$. Para a comparação de médias, utilizou-se o teste de Scoot-
Knott, a 5\% de probabilidade. Efetuou-se também uma análise de regressão para os dados de floração e massa dos frutos.

\section{RESULTADOS E DISCUSSÃO}

\section{Retardamento da floração natural}

$\mathrm{Na}$ primeira avaliação do processo de floração do abacaxizeiro 'Pérola', constatou-se que a aplicação de $30 \mathrm{mg} \mathrm{L}^{-1}$ de diquat, com e sem ureia, determinou um percentual de 90,2 e $85,6 \%$ de inibição do florescimento, significativamente maior que os demais tratamentos (Tabela 2). A concentração de $23 \mathrm{ml} \mathrm{L}^{-1}$ de diquat $+20 \mathrm{~g} \mathrm{~L}^{-1}$ de ureia apresentou um percentual de inibição de $63,2 \%$, significativamente maior que a mesma concentração sem ureia $(40,6 \%)$ e os demais tratamentos. A testemunha apresentava, nesta data apenas $23,6 \%$ de plantas que não apresentavam a inflorescência, ou seja, 76,4\% haviam florescido. Nas avaliações seguintes de 15 de setembro e 10 de outubro, as tendências foram as mesmas.

A evolução dos índices de inibição da floração também foi constatada através da construção das equações de regressão apresentada na Tabela 5. A concentração máxima ( $\left.30 \mathrm{mg} \mathrm{L}^{-1}\right)$ de diquat dentro do desdobramento sem ureia, avaliada em 20 de agosto, controlou a floração em 77,9\%, índice muito acima da testemunha, que foi de $17,7 \%$. A maior concentração de diquat dentro do desdobramento com ureia produziu um controle ainda mais elevado da floração, atingindo 88,6\%, enquanto a menor concentração testada, de $15 \mathrm{mg} \mathrm{L}^{-1}$, controlou a floração em 49\%. Nas avaliações de 15 de setembro e 10 de outubro, as tendências foram as mesmas, apenas os índices de florescimento foram aumentando, ou seja, na medida em que o tempo passa, mais plantas entram em processo de floração, sugerindo que o fitorregulador apenas retarda a floração, tendo um prazo de ação determinado. A comparação do tratamento com $30 \mathrm{mg} \mathrm{L}^{-1}$ diquat + ureia, com a mesma concentração sem ureia, indica possivelmente que a ureia potencializou a ação do diquat, pois onde esta estava presente, a floração natural foi menor em, aproximadamente, $14 \%, 35 \%$ e $35 \%$ nas três datas de avaliação.

Estes resultados demonstram que a utilização do diquat pode atrasar a floração. A diferenciação floral do abacaxizeiro é uma resposta fisiológica à elevação do teor de etileno no meristema apical. (BURG; BURG, 1966). Como o etileno é produzido a partir da ACC, através da enzima ACC oxidase, presente em altas concentrações na fase reprodutiva do abacaxizeiro, provavelmente o diquat tenha inibido a síntese da ACC oxidase que, por sua vez, 
reduziu o etileno, retardando a diferenciação floral. Para Antunes et al. (2008), o Paclobutrazol inbiu a diferenciação floral natural do abacaxizeiro 'Smooth cayenne', enquanto Sampaio et al. (1998) não encontraram esta resposta testando o ácido alfanaftaleno acético (ANA), nas concentrações e condições estudadas. Barbosa et al. (1998), avaliando o efeito na diferenciação floral natural de inibidores de crescimento à base de ácido 2-(3-clorofenoxi) propiônico, cloreto de mepiquat, ureia líquida e sólida e paclobutrazol, encontraram resposta significativa apenas para este último que, a 100 ppm, inibiu até $82,22 \%$ da floração no abacaxizeiro 'Pérola'.

Na região onde foi conduzido o experimento, as condições climáticas favoráveis ao processo de diferenciação floral (redução de temperatura, fotoperíodo e precipitação) ocorrem nos meses de abril a agosto (Tabela1), dificultando o manejo da cultura. Como consequência, ocorre uma sazonalidade de produção, com pico de colheita em novembro e dezembro, causando sérios transtornos aos produtores desta cultura e à indústria instalada na região, que fica ociosa em grande parte do ano. Neste sentido, os resultados obtidos neste trabalho, indicando a possibilidade de retardar a diferenciação floral evitando a floração natural, revestem-se de fundamental importância, uma vez que a colheita poderá ser realizada em períodos mais amplos ao longo do ano.

\section{Massa do fruto e desenvolvimento da planta}

A avaliação da massa fresca do fruto evidenciou que os tratamentos com a maior concentração de diquat $\left(30 \mathrm{ml} \mathrm{L}^{-1}\right)$, com e sem ureia, apresentaram redução de 113 e 172 gramas em relação à testemunha, enquanto os demais não diferiram significativamente (Tabela 3). Esta redução no tamanho dos frutos talvez seja devida aos efeitos fitotóxicos do diquat observados inicialmente nas folhas. Entretanto, desdobrando-se as concentrações de diquat com e sem ureia, através da análise de regressão (tabela 5), constatou-se que a massa do fruto, no caso da concentração máxima do diquat sem ureia, foi de $1.001 \mathrm{~g}$ e, na testemunha, foi de $1.116 \mathrm{~g}$, reduzindo, portanto, apenas $15 \mathrm{~g}$, enquanto a concentração máxima do diquat $\left(30 \mathrm{ml} \mathrm{L}^{-1}\right)$ com ureia reduziu o tamanho do fruto de $1.075 \mathrm{~g}$ para $957 \mathrm{~g}$, ou seja, uma redução de $118 \mathrm{~g}$ em relação ao tratamento com 15 $\mathrm{ml} \mathrm{L}^{-1}$. Estes resultados indicam que o uso da ureia contribuiu ainda mais para a redução da massa do fruto. Este resultado pode estar associado ao fato de que o nitrogênio presente na ureia determina maior abertura estomática ou afastamento das plaquetas de cera, ocasinando maior absorção do regulador por via simplástica na folha e, assim, a ação do diquat tornou-se mais acentuada.

A utilização da concentração máxima do diquat sem ureia determinou um aumento na massa, e consequentemente, no tamanho da coroa. A folha " $D$ " apresentou-se mais larga na testemunha, enquanto os outros parâmetros estudados, massa e número de filhotes, comprimento da folha " $D$ " e sólidos solúveis ( ${ }^{\circ}$ Brix), não apresentaram diferença significativa em decorrência da aplicação dos tratamentos (Tabelas 3 e 4$)$.

Efeitos fitotóxicos foram visualmente constatados nas folhas, com o aparecimento de manchas sintomáticas, logo após a aplicação dos tratamentos, mas que, no entanto, recuperaram-se com o decorrer do tempo através da emissão de novas folhas. $\mathrm{O}$ diquat é um inibidor de reduzida translocação, que atua no fotossistema I, muito próximo ou mesmo na ferredoxina, capturando elétrons provenientes da fotossíntese e através de um processo de auto-oxidação, transforma os radicais tóxicos em superóxidos, que sofrem o processo de dismutação, formando peróxido de hidrogênio responsável pela degradação das membranas (peroxidação de lipídios), ocasionando vazamento do suco celular, necrosamento e morte dos tecidos. Algumas espécies, entretanto, parecem ter desenvolvido um mecanismo de resistência, através da destoxificação dos radicais superóxidos pelas enzimas superóxido-dismutase, catalase e peroxidase. Estas plantas podem evidenciar sinais de fitotoxidade, mas recuperam-se após certo tempo, uma vez que não apresentam translocação, o que parece ser o caso do abacaxizeiro. O comprimento e a largura da folha "D", a massa e o número de filhotes e a qualidade dos frutos, através da leitura dos sólidos solúveis totais ( ${ }^{\circ}$ brix) do suco, não foram comprometidos. Entretanto, como a massa dos frutos foi reduzida nas maiores dosagens do diquat, recomenda-se que mais estudos sejam realizados. Antunes et al. (2008), testando o Paclobutrazol, relatam alterações morfológicas através do fechamento da roseta foliar devido ao enrolamento e a torção das folhas jovens, que no entanto não causaram maiores danos as plantas $\mathrm{e}$ produziram frutos normais. Barbosa et al. (2003) e Rebolledo et al. (1997) também mencionam a ocorrência de uma torção na roseta foliar quando utilizaram o ácido 2-(3-clorofenoxi) propiônico (ACP), mas estes últimos autores fazem referência a uma elevada mortalidade de plantas, quando usaram $200 \mathrm{mg} \mathrm{L}^{-1}$. Min e Bartholomew (1993) relatam que o CPA (Fruitone), na concentração de $2,5 \mathrm{mg}$ por planta, causou a morte de algumas plantas mais jovens, enquanto a dosagem de $0,5 \mathrm{mg}$ por planta provocou apenas uma 
leve torção das folhas do abacaxizeiro.

TABELA 1 - Temperaturas mínimas e máximas mensais $\left({ }^{\circ} \mathrm{C}\right)$ e precipitação mensal $(\mathrm{mm})$ na região do Campo experimental da EMPAER. (Estação Meteorológica da UNEMAT, Tangará da Serra, 2007/2008).

\begin{tabular}{lccc}
\hline Mes/ano & Temp. Mín. $\left({ }^{\circ} \mathrm{C}\right)$ & Temp. Máx. $\left({ }^{\circ} \mathrm{C}\right)$ & Precipitação $(\mathrm{mm})$ \\
\hline Abril/07 & 19,7 & 29,5 & 118 \\
Maio/07 & 17,9 & 28,0 & 37 \\
Junho/07 & 16,7 & 27,9 & 3 \\
Julho/07 & 17,8 & 32,6 & 0 \\
Agosto/07 & 19,5 & 34,6 & 32 \\
Setembro/07 & 19,2 & 33,6 & 138 \\
Outubro/07 & 20,9 & 33,0 & 171 \\
Novembro/07 & 21,0 & 32,2 & 181 \\
Dezembro/07 & 21,6 & 31,1 & 301 \\
Janeiro/08 & 21,7 & 30,9 & 329 \\
Fevereiro/08 & 21,3 & 30,3 & 435 \\
Março/08 & 21,5 & 30,5 & 189 \\
Abril/08 & 17,7 & 29,4 & 181 \\
Maio/08 & 16,8 & 28,5 & 20 \\
Junho/08 & 16,1 & 30,6 & 6 \\
Julho/08 & 16,5 & 31,6 & 3 \\
Agosto/08 & 17,1 & 32,1 & 48 \\
\hline
\end{tabular}

TABELA 2 - Percentual de inibição da floração no abacaxizeiro 'Perola', em função da aplicação dos tratamentos. UFLA, Lavras, 2010.

Datas de avaliação de floração

\begin{tabular}{llcc}
\cline { 2 - 4 } Trat. & 20 de agosto & 15 de setembro & 10 de outubro \\
\hline $0 \mathrm{ml} \mathrm{L}^{-1}$ diquat (test.) & $23,6 \mathrm{c}$ & $18,8 \mathrm{~b}$ & $12,9 \mathrm{~b}$ \\
$15 \mathrm{ml} \mathrm{L}^{-1}$ diquat & $31,9 \mathrm{c}$ & $19,2 \mathrm{~b}$ & $14,4 \mathrm{~b}$ \\
$15 \mathrm{ml} \mathrm{L}^{-1}$ diquat $+20 \mathrm{~g} \mathrm{~L}^{-1}$ & $51,1 \mathrm{c}$ & $30,2 \mathrm{~b}$ & $25,4 \mathrm{~b}$ \\
$23 \mathrm{ml} \mathrm{L}^{-1}$ diquat & $40,6 \mathrm{c}$ & $23,6 \mathrm{~b}$ & $22,7 \mathrm{~b}$ \\
$23 \mathrm{ml} \mathrm{L}^{-1}$ diquat $+20 \mathrm{~g} \mathrm{~L}^{-1}$ & $63,2 \mathrm{~b}$ & $41,7 \mathrm{~b}$ & $33,5 \mathrm{~b}$ \\
$30 \mathrm{ml} \mathrm{L}^{-1}$ diquat & $85,4 \mathrm{a}$ & $70,6 \mathrm{a}$ & $64,8 \mathrm{a}$ \\
$30 \mathrm{ml} \mathrm{L}^{-1}$ diquat $+20 \mathrm{~g} \mathrm{~L}^{-1}$ & $90,2 \mathrm{a}$ & $85,6 \mathrm{a}$ & $81,3 \mathrm{a}$ \\
\hline $\mathrm{CV}(\%)$ & 12,6 & 18,1 & 18,9 \\
\hline
\end{tabular}

Médias seguidas de mesma letra na coluna não diferem significativamente entre si, pelo teste de Scott-Knott, a 5\% de probabilidade.

TABELA 3 - Massa do fruto, da coroa, dos filhotes e número de filhotes do abacaxizeiro 'Pérola', em função da aplicação dos tratamentos. UFLA, Lavras, 2010.

\begin{tabular}{lcccc}
\hline & $\begin{array}{c}\text { fruto } \\
\text { (gramas) }\end{array}$ & $\begin{array}{c}\text { coroa } \\
\text { (gramas) }\end{array}$ & $\begin{array}{c}\text { filhote } \\
\text { (gramas })\end{array}$ & $\begin{array}{c}\mathrm{n}^{\text {o }} \text { filhotes } \\
\text { (Unidade) }\end{array}$ \\
$0 \mathrm{ml} \mathrm{L}$ diquat (test.) $_{15 \mathrm{ml} \mathrm{L}^{-1} \text { diquat }}^{1.109,3 \mathrm{a}}$ & $117 \mathrm{~b}$ & $197 \mathrm{a}$ & $9,7 \mathrm{a}$ \\
$15 \mathrm{ml} \mathrm{L}^{-1}$ diquat $+20 \mathrm{~g} \mathrm{~L}^{-1}$ & $1.094,0 \mathrm{a}$ & $107 \mathrm{~b}$ & $190 \mathrm{a}$ & $11,2 \mathrm{a}$ \\
$23 \mathrm{ml} \mathrm{L}^{-1}$ diquat & $1.050,3 \mathrm{a}$ & $112 \mathrm{~b}$ & $166 \mathrm{a}$ & $11,3 \mathrm{a}$ \\
$23 \mathrm{ml} \mathrm{L}^{-1}$ diquat $+20 \mathrm{~g} \mathrm{~L}^{-1}$ & $1.054,0 \mathrm{a}$ & $118 \mathrm{~b}$ & $192 \mathrm{a}$ & $7,7 \mathrm{a}$ \\
$30 \mathrm{ml} \mathrm{L}^{-1}$ diquat & $1.066,8 \mathrm{a}$ & $110 \mathrm{~b}$ & $191 \mathrm{a}$ & $8,7 \mathrm{a}$ \\
$30 \mathrm{ml} \mathrm{L}^{-1}$ diquat $+20 \mathrm{~g} \mathrm{~L}^{-1}$ & $996,3 \mathrm{~b}$ & $153 \mathrm{a}$ & $187 \mathrm{a}$ & $12,2 \mathrm{a}$ \\
\hline $\mathrm{CV}(\%)$ & $937,0 \mathrm{~b}$ & $127 \mathrm{~b}$ & $175 \mathrm{a}$ & $7,0 \mathrm{a}$ \\
\hline
\end{tabular}

Médias seguidas de mesma letra na coluna não diferem significativamente entre si, pelo teste de Scott-Knott, a 5\% de probabilidade. 
TABELA 4 - Comprimento e largura da folha ' $\mathrm{D}$ ' (cm) e sólidos solúveis ( ${ }^{\circ}$ Brix) do suco do abacaxizeiro 'Pérola', em função da aplicação dos tratamentos. UFLA, Lavras, 2010.

\begin{tabular}{|c|c|c|c|}
\hline Tratamentos & $\begin{array}{c}\text { Comprimento } \mathrm{fl} \text { ' } \mathrm{D} \text { ' } \\
(\mathrm{cm})\end{array}$ & $\begin{array}{l}\text { Largura fl. 'D' } \\
\text { (cm) }\end{array}$ & $\begin{array}{l}\text { Sólidos } \\
\text { soluveis } \\
\text { ('brix) }\end{array}$ \\
\hline $0 \mathrm{ml} \mathrm{L}{ }^{-1}$ diquat (test.) & $111,3 \mathrm{a}$ & $6,8 \mathrm{a}$ & $13,75 \mathrm{a}$ \\
\hline $15 \mathrm{ml} \mathrm{L}^{-1}$ diquat & $109,0 \mathrm{a}$ & $5,8 \mathrm{~b}$ & $13,40 \mathrm{a}$ \\
\hline $15 \mathrm{ml} \mathrm{L}^{-1}$ diquat $+20 \mathrm{~g} \mathrm{~L}^{-1}$ & $106,9 \mathrm{a}$ & $5,6 \mathrm{~b}$ & $14,00 \mathrm{a}$ \\
\hline $23 \mathrm{ml} \mathrm{L}^{-1}$ diquat & $108,6 \mathrm{a}$ & $5,3 \mathrm{~b}$ & $13,80 \mathrm{a}$ \\
\hline $23 \mathrm{ml} \mathrm{L}^{-1}$ diquat $+20 \mathrm{~g} \mathrm{~L}^{-1}$ & $104,8 \mathrm{a}$ & $5,5 \mathrm{~b}$ & $14,20 \mathrm{a}$ \\
\hline $30 \mathrm{ml} \mathrm{L}^{-1}$ diquat & $109,7 \mathrm{a}$ & $5,1 \mathrm{~b}$ & $13,90 \mathrm{a}$ \\
\hline $30 \mathrm{ml} \mathrm{L}^{-1}$ diquat $+20 \mathrm{~g} \mathrm{~L}^{-1}$ & $118,7 \mathrm{a}$ & $5,6 \mathrm{~b}$ & $13,30 \mathrm{a}$ \\
\hline $\mathrm{CV}(\%)$ & 8,77 & 12,13 & 7,74 \\
\hline
\end{tabular}

Médias seguidas de mesma letra na coluna não diferem significativamente entre si, pelo teste de Scott-Knott, a 5\% de probabilidade.

TABELA 5 - Inibição da floração determinada por doses do diquat, sem e com uréia, em três avaliações, e massa dos frutos do abacaxizeiro 'Pérola'. UFLA, Lavras, 2010.

\begin{tabular}{llccc}
\hline Variáveis & $\begin{array}{c}\text { Modelo reduzido } \\
\text { sequencial de regressão }\end{array}$ & $\mathrm{R}^{2}(\%)$ & $\begin{array}{c}\text { dose máx. } \\
\text { diquat }\end{array}$ & $\begin{array}{c}\text { dose mín. } \\
\text { diquat }\end{array}$ \\
\hline
\end{tabular}

\begin{tabular}{|c|c|c|c|c|}
\hline \multicolumn{5}{|c|}{ Diquat dentro do desdobramento sem ureia } \\
\hline Inibição em 20/agosto & $Y=0,066802+17,733575 x^{2}$ & 89,12 & 77,9 & 17,7 \\
\hline Inibição em 15/setembro & $Y=0,056308+9,747822 x^{2}$ & 76,25 & 60,4 & 9,8 \\
\hline Inibição em 10/outubro & $Y=0,057173+5,065398 x^{2}$ & 83,23 & 56,5 & 5,1 \\
\hline Massa do fruto & $Y=-0,1281+1.116,3433 x^{2}$ & 98,10 & $1.001,0$ & $1.116,0$ \\
\hline \multicolumn{5}{|c|}{ Diquat dentro do desdobramento com ureia } \\
\hline Inibição em 20/agosto & $Y=0,058579+35,845285 x^{2}$ & 97,46 & 88,6 & 49,0 \\
\hline Inibição em 15/setembro & $Y=0,083429+6,502842 x^{2}$ & 92,94 & 81,6 & 25,3 \\
\hline Inibição em 10/outubro & $Y=0,084369+0,217935 x^{2}$ & 89,40 & 76,2 & 19,2 \\
\hline Massa do fruto & $Y=-0,1744+1.114,1381 x^{2}$ & 69,65 & 957,0 & $1.075,0$ \\
\hline
\end{tabular}

\section{CONCLUSÕES}

Na região onde foi conduzido o experimento, $\mathrm{o}$ processo de diferenciação floral natural do abacaxizeiro 'Pérola', que ocorre de abril a agosto, foi evitado. A ação do diquat na concentração de $30 \mathrm{ml} \mathrm{L}-^{1}$ retardou o florescimento, muito embora tenha reduzido a massa dos frutos. A ureia potencializou a ação do diquat no retardamento do florescimento. Estes resultados permitem que o período de colheita seja alongado, beneficiando os produtores e a indústria.

\section{AGRADECIMENTOS}

Os autores agradecem à Empresa Tropical Polpa de Frutas Tangará Ltda, pela contribuição em insumos e mão de obra cedidos; aos técnicos agrícolas da EMPAER-MT Moacir Buffete (in memoriam) e Adair Osvino Franke, pela colaboração na aplicação dos tratamentos e coleta de dados; e a UNEMAT, pelo fornecimento dos dados meteorológicos. 


\section{REFERÊNCIAS}

AGRIANUAL. Anuário da agricultura brasileira. São Paulo: FNP, 2009.p.144-152.

ANTUNES, A.M.; ONO, E.O.; SAMPAIO, A.C. Efeito do Paclobutrazol no controle da diferenciação floral natural do abacaxizeiro cv. Smooth cayenne.

Revista Brasileira de Fruticultura, Jaboticabal, v.30, n.2, p.290-295, 2008.

BARBOSA, N.M.L.; CUNHA, G.A.P. da; REINHARDT, D.H.; BARROS, P.G. Controle da floração natural do abacaxizeiro 'Pérola' com ureia e reguladores de crescimento, no Recôncavo Baiano. Revista Brasileira de Fruticultura, Jaboticabal, v.20, n.3, p.359-366, 1998.

BARBOSA, N.M.L.; CUNHA, G.A.P. da; REINHARDT, D.H.; BARROS, P.G.; SANTOS, A.R.L. dos. Indução de alterações morfológicas e anatômicas em folhas de abacaxizeiro 'Pérola' pelo ácido 2-(3-clorofenoxi) propiônico. Revista Brasileira de Fruticultura, Jaboticabal, v.25, n.3, p.386-389, 2003.

BURG, S.P.; BURG, E.A. Auxin-induced ethylene formation and its relation to flowering in the pineapple. Science, Washington, v.152, p.1.269, 1966.

CUNHA, G.A.P. da; CABRAL, J.R.S.; SOUZA, L.F. da S. (Org.). O Abacaxizeiro: cultivo, agroindústria e economia. Brasília: Embrapa, 1999. 480p.

CUNHA, G.A.P. da; COSTA, J.T.A.; REINHARDT, D.H. Natural flowering in pineapple: inhibition by growth regulators. Fruits, Paris, v.58, n.1, p.27-37, 2003.

MIN, X.J.; BARTHOLOMEW, D.P. Effects of growth regulators on ethylene production and floral initiation of pineapple. Acta Horticulturae, Wageningen, n.334, p.101-112, 1993.
NICKELL, L.G. Plant growth substances. In: KIRHOTHMER. Encyclopedia of chemical technology. Hoboken: John Wiley \& Sons, 1982. v.18, p.1-23.

ORIOLI, A.L. Levantamento detalhado dos solos do campo experimental de Tangará da SerraMT. Cuiabá. EMPA/MT, 1985. 79 p. (Boletim de Pesquisa, 2).

RABIE, E.C.; TUSTIN, H.A.; WESSON, K.T. Inhibition of natural flowering occurring during the winter months in queen pineapple in Kwazulu Natal, South Africa. Acta Horticulturae, The Hague, n.529, p.175-183, 2000.

REBOLLEDO, A.M.; URIZA, D.E.A.; AGUIRRE, L.A.G.; PAPALOAN, C.E. Inhibition de la floración de la pinã con diferentes dosis de Fruitone CPA em dos densidades de siembra. Acta Horticulturae, The Hague, n.425, p.347-354, 1997.

SAMPAIO, A.C.; FUMIS, T. de F.; HERNANDES V.A. de N. Ácido alfanaftalenoacético (ANA) no controle da diferenciação floral natural do abacaxizeiro Cv. Smooth cayenne. Revista Brasileira de Fruticultura, Jaboticabal, v.20, n.3, p.353-358, 1998.

SOUZA, M. de; GUIMARÃES, P.T.G.; CARVALHO, J.G. de; FRAGOAS, J.C. Sugestões de adubação para plantas frutíferas: Abacaxizeiro. In: RIBEIRO, A.C.; GUIMARÃES, P.T.G.; ALVAREZ, V.H.V. (Ed.). Recomendações para o uso de corretivos e fertilizantes em Minas Gerais. $5^{\text {a }}$ Aproximação. Viçosa: UFV, Comissão de Fertilidade do Solo do Estado de Minas Gerais, 1999. p.209-216.

WANG, S.Y.; STEFFENS, G.L. Effect of paclobutrazol on water stress-induced ethylene biosynthesis and polyamine accumulation in apple seedling leaves Phytochemistry, New York, v.24, n.10, p.2.1852.190, 1985. 